\title{
Les montagnes du Pacifique de Dominique CADILHAC
}

\section{Hélène Guiot}

\section{(2) OpenEdition}

1 Journals

Édition électronique

URL : http://journals.openedition.org/jso/6337

DOI : $10.4000 /$ jso. 6337

ISSN : $1760-7256$

\section{Éditeur}

Société des océanistes

\section{Édition imprimée}

Date de publication : 30 juin 2011

Pagination : 202-203

ISBN : 978-2-85430-030-7

ISSN : 0300-953x

Référence électronique

Hélène Guiot, «Les montagnes du Pacifique de Dominique caDILHAC », Journal de la Société des Océanistes [En ligne], 132 | 1er semestre 2011, mis en ligne le 30 juin 2011, consulté le 24 septembre 2020. URL http://journals.openedition.org/jso/6337 ; DOI : https://doi.org/10.4000/jso.6337 
Cadilhac Dominique, 2007. Les montagnes du Pacifique, 160 p., table des 100 objets reproduits, bibliogr. indicative, cartes, nombreuses ill. couleur.

Louvrage de Dominique Cadilhac emprunte son titre à la configuration des îles hautes et escarpées des Marquises, où il séjourna en tant qu'administrateur d'État de 1994 à 1996. Nourri de son expérience et des souvenirs personnels, il nous livre ici un roman dans lequel il donne la parole à un médecin, Christian. "Voileux tourdumondiste", ce dernier fait escale aux Marquises et y demeure finalement quelques années comme docteur de l'archipel. Le récit de ses tournées médicales dans les différentes îles, parfois au plus profond des vallées, sont pour l'auteur l'occasion de donner la parole à de multiples acteurs, polynésiens et occidentaux, installés ou de passage, tout en dressant un tableau de l'atmosphère sociale et économique des îles Marquises au tournant des années 2000.

Dominique Cadilhac émaille ainsi le récit de rencontres qui permettent au personnage principal et, par là-même, on le suppose, à l'auteur, d'exposer son ressenti vis-à-vis des Marquisiens et de développer plusieurs sujets récurrents au gré des dix-neuf chapitres du roman : l'évolution des modes de vie dans ces milieux insulaires, la place des femmes dans les sociétés de Polynésie, les rapports entre les êtres humains et la communication entre les communautés, la colonisation, l'oral et l'écrit, la vie sentimentale et sexuelle des habitants, la violence, l'isolement, l'histoire de l'archipel marquisien... et bien d'autres encore.

C'est d'ailleurs un monde de ruines et de chute démographique que décrit souvent l'auteur : vestiges archéologiques des habitats occupés avant l'arrivée des Occidentaux, ruines de l'époque où la production du coprah constituait le revenu de nombreuses familles marquisiennes, avant la mise en route du CEP et l'exode qui s'ensuivit vers Tahiti ; l'arrivée des Européens, encore, qui introduisirent des maladies contre lesquelles les insulaires n'étaient pas protégés, ainsi que des armes à feu générant conflits d'intérêt et, aussi, décès.

$\mathrm{Au}$ fil de l'histoire, on reconnaît aisément des personnalités de ces îles Marquises, parmi lesquelles se mêlent des personnages inventés et d'autres recréés par l'auteur, combinant alors souvenirs personnels et fiction. Ainsi défilent un grand nombre d'acteurs que l'on revoit ou non au détour des pages, donnant un aspect légèrement décousue à l'histoire; mais c'est peut-être un effet recherché par l'auteur qui veut ainsi rendre comte du « simple passage » de son héros, comme bien d'autres visiteurs, dans ces îles. On regrettera d'ailleurs quelques erreurs, comme l'or- thographe approximative des termes polynésiens (kaoha, Raïatea...), les deux références bibliographiques incomplètes (Testard de Marans ${ }^{1}$ p. 184 et Albert Cohen p. 211), ou bien encore "Nuku Hiva, baptisée la terre des hommes » (p. 46) alors que l'expression (en marquisien henua enana ou fenua enata) désigne l'ensemble de l'archipel. Les lecteurs accèderont souvent aux pensées répétées du médecin (celles de l'auteur, celles entendues par lui lors de son séjour ?) sur la solitude, l'isolement, le silence qui s'abat sur ces terres, ou encore sur la majesté du paysage et les grandes montagnes qui forgent le caractère des insulaires. On restera d'ailleurs prudent lorsque ces pensées expliquent à quel point le milieu conditionne le caractère des habitants des Marquises. Ainsi, à Ua Pou, où la géologie paraît différente, « une île plus facile à habiter où l'homme semble se plaire ", "les Marquisiens ... sont moins marqués par la gravité de caractère si présente chez leurs autres compatriotes [les habitants des autres îles de l'archipel], et plus résolument chaleureux dans leurs comportements" (p. 242). De même, le médecin, tentant de décrypter les comportements contemporains, voire la psychologie des Marquisiens, s'attèle à une tâche délicate et dans laquelle il ne parvient pas toujours à convaincre.

Au-delà de ces réserves, l'auteur propose des analyses intéressantes sur l'importance des cycles du vivant (p. 111), sur l'engouement pour le passé ancien des îles Marquises, qui tend à évincer la connaissance de l'histoire plus récente (p. 130). Il décrit avec justesse la douleur que provoque le travail de retour sur ce passé (p. 226), parce que rien, ou si peu, a été transmis, en raison notamment de l'interdiction de parler la langue marquisienne. Il fait expliquer par une Marquisienne de son roman le travail de copiste que constituait à l'école l'apprentissage de la langue française (p. 213). Si elle reconnait que l'usage du français lui ouvre des portes, il reste fermé, pour elle, à l'expression de l'émotion. Enfin, et peut-être surtout, elle déclare: "nous avons perdu l'usage d'une pensée active faute d'une langue élaborée, vivante, entretenue".

Enfin et, c'est peut-être le plus grand intérêt de ce roman, l'auteur présente les débuts et l'ascension d'un leader politique marquisien, que l'on devine être Lucien Kimitete.

Lucien Kimitete (1952-2002) fut maire de Nuku Hiva, conseiller à l'assemblée territoriale de Polynésie française et numéro deux du parti polynésien autonomiste d'opposition, le Fetia Api. Engagé politiquement et ardent militant de la spécificité culturelle marquisienne, Lucien Kimitete luttait justement contre les a priori des visiteurs qui voient trop souvent les Marquises comme un cimetière. Animateur charismatique, 
notamment du festival des Marquises (qui se tient tous les quatre ans et dont le prochain aura lieu à Nuku Hiva en décembre 2011), il disparaît dans un accident d'avion, en mai 2002, au large des Tuamotu, où il se rendait dans le cadre d'une campagne pour des élections législatives, avec ses compagnons d'infortune, Boris Leontieff, Arsen Tuairau et Ferfine Besseyre ${ }^{2}$. Les conditions de l'accident restant insuffisamment expliquées, la douleur de l'absence fut encore plus vivement ressentie aux îles Marquises :

«Kia [Lucien Kimitete] n'est pas mort, non, pire, il a disparu et son corps absent ne peut nous guérir de son souvenir. » (p. 266)

L'auteur évoque alors une certaine suspicion qui circulera parmi la population:

"le moment où elle [la disparition] se produit, dans l'existence de Kia et de tous ceux qui le connaissent, accroît le trouble dans lequel elle va plonger beaucoup d'esprits. Elle intervient dans un contexte bien opportun pour ceux que son action et ses discours étaient parvenus à inquiéter. » (p. 266)

Le roman se poursuit et tout au long de ce récit, reflet d'un grand attachement aux Marquisiens, ceux des lecteurs qui ont séjourné quelque temps dans cet archipel, verront s'animer des personna lités connues; ils revivront peut-être certains des événements relatés et éprouverons assurément des impressions familières.

Hélène Guiot, UMR 7041 ArScAn - Nanterre

Colombo Dougoud Roberta et Barbara Müller (s. d.), 2010. Traces de rêves. Peintures sur écorce des Aborigènes d'Australie, Gollion-Genève, Infolio éditions-Musée d'Ethnographie de Genève, 176 p., bibliogr., glossaire, cartes, nombreuses ill.

Venant après le bel ouvrage sur les Bambous kanak, une passion de Marguerite Lobsinger-Dellenbach (décrit dans le JSO 126-127), ce nouveau volume de la collection "Sources et témoignages » du MEG restera lui aussi non pas seulement comme le guide très détaillé et illustré d'une exposition elle-même excellemment conçue (présentée à Genève du 17 septembre 2010 au 27 février 2011), mais comme un livre indispensable dans son domaine. Quoique présentes dans de très nombreux musées, les écorces peintes des artistes des communautés aborigènes du nord de l'Australie n'ont suscité que fort peu de publications illustrées et Traces de rêves (également disponible en version anglaise, Dream Traces) comble une lacune spécialement pour les lecteurs francophones, qui jusqu'à présent ne disposaient guère que des livres anciens de Kupka (1962 et 1972). Surtout, si l'intention déclarée était de proposer « un b.a.-ba de l'art aborigène produit en Terre d'Arnhem, une initiation destinée à permettre la reconnaissance et la lecture d'un corpus plastique déterminé" comme l'écrit le directeur du MEg Boris Wastiau (p. 7), ce livrecatalogue va largement au-delà de cette modeste ambition en mettant à la portée des lecteurs les connaissances les plus récentes avec une clarté qui ne se dément jamais.

Les 74 peintures sur écorce exposées occupent le centre de l'ouvrage (pp. 43-117), encadré par deux séries d'articles venant les éclairer. La sélection de ces œuvres a obéi à deux principes, montrer au public l'ensemble de la collection actuelle du MEG ( 57 peintures) et l'ordonner par thèmes, en la complétant au besoin par des emprunts à d'autres musées suisses, celui de Bâle en particulier. Cette présenta tion thématique (Eau, terre et ciel; Mythes; Vie profane, vie sacrée; Esprits; Tiwi ; Bestiaire; Du rêve au monde extérieur), introduit efficacement les visiteurs dans l'univers de ces peintures, tout en étant infiniment plus conforme à leur esprit et à celui de leurs créateurs que tous les classements avancés jusqu'à présent en Occident, par nom d'artistes, clan, origine géographique, etc. De la sorte, il n'est plus question de continuer à distinguer avec Kupka (1962) entre "peinture figurative, peinture symbolique, peinture ornementale", puis entre "symbolisme figuratif " et "symbolisme "abstrait" ", ou de séparer les peintures cérémonielles et les écorces peintes "séculières", comme le faisait Mountford, tout en ébauchant un classement thématique (1958 : 160-169).

Pour beaucoup de ces peintures, un tel classement dépend entièrement des interprétations de leur disposition et de leurs motifs, recueillies auprès de leurs créateurs ou de leurs exégètes les plus proches. Dans cette perspective, une autre particularité remarquable de cette exposition et de ce catalogue est de s'être assuré le concours de l'un d'eux, Joe Neparrnga Gumbula, ancien du clan Gupanuyngu des Yolngu, sur la côte orientale de la Terre d'Arnhem, impliqué depuis longtemps dans l'appréciation et l'identification des écorces peintes, et que le MEG avait déjà sollicité à ce titre en 2006. Ce ne sont pas seulement les notices ou l'ordonnancement thématique des œuvres qui ont bénéficié de sa collaboration, mais l'architecture même du catalogue, dont on aura tout intérêt à suivre le parcours. L'avant-propos de Boris Wastiau, le texte de Joe Gumbula ("Les peintures sont l'ossature de la terre et de la mer"), ceux de Jessica de Largy Healy ("Ces peintures nous disent qui nous sommes": les fondations de l'art aborigène du nord de l'Australie» et "Matières et couleurs ») 